\title{
Ruptured Ectopic Pregnancy: A Case of Fair Complexion Woman at Tamale Teaching Hospital, Ghana
}

\author{
Asare HK*, Waliwu D and Adu-Ntow D \\ Department of Radiology, Tamale Teaching Hospital, \\ Ghana \\ *Corresponding author: Asare HK, Department of \\ Radiology, Tamale Teaching Hospital, Ghana
}

Received: November 05, 2017; Accepted: November 29, 2017; Published: December 06, 2017

\begin{abstract}
The incidence of Ectopic Pregnancy (EP) is rising globally and as such in Ghana. In Ghana, the observation is that, EP is associating significantly with women of fair skin complexion, whether is artificially induced or natural. Our recent case finding at the Tamale Teaching Hospital (TTH) in Ghana compares well with previous research of other hospital in the country. Early diagnoses improve patient outcome with ultrasound scan been useful diagnostic tool. The sonographic features observed with our case finding were; free fluid in peritoneum spaces, large right complex adnexal mass and bulky uterus with slight texture irregularities. The case is presented below.
\end{abstract}

Keywords: Ectopic pregnancy; Fair woman; Ultrasound; Africa

\section{Case Presentation}

A 25 year old woman, gravida 2 para 2, fair in skin complexion presented at the gynecology emergency clinic of the Tamale Teaching Hospital (TTH) with complains of lower abdominal pain and 3days of scanty vaginal bleeding. Rigorous clinical examination revealed pain at supra-pelvic region on palpation, minimal pain at iliac fossa regions, weakness, dizziness and positive nausea but no significant dilated cervix OS $(0.5 \mathrm{~cm})$. Patient has previous history of using oral contraceptive pills for a year and sexual affair with three multiple partners. However, she has no history of pelvic inflammatory disease, ectopic pregnancy, assisted technology fertilization and tubal surgery. Patient was referred to the radiology department for abdominal-pelvic ultrasound scan to rule out Ectopic Pregnancy (EP) or threatened abortion. Until this, she had tested positive on Urine Pregnancy Test (UPT) 4wks after her last menstrual period. However, earlier ultrasound scan following the UPT ruled out any evidence of cyesis at a different facility other than TTH. At the TTH radiology department, the ultrasound scan performed diagnosed the case as ruptured EP. The sonographic features observed with our case finding were; free fluid in peritoneum spaces, large right complex adnexal mass (measuring $27.1 \mathrm{~cm}$ in circumference) and bulky uterus with slight texture irregularities as shown in (Figure 1). The diagnosis was confirmed at laparotomy as right ruptured EP's.

\section{Discussion}

Ectopic Pregnancy (EP) refers to the implantation of a fertilized egg or fetus in any site other than the uterine cavity. In simple term, $\mathrm{EP}$ is an abnormal pregnancy complication in which the product of conception develops outside the uterine cavity, and mostly not viable except in rare cases [1]. Consequently, the abnormally implanted gestation grows and when it enlarges create the potential for organ rupture. Due that the uterus is the only cavity that can expand and accommodate fetal development or growth [1]. EP is a worldwide health burden, and by far one of the commonest critical and lifethreatening surgical emergencies among women of reproductive age. It is thus considered the most cause of maternal morbidity and mortality (73\%) in early pregnancy [2,3]. The evidence suggests that, incidence of EP has increased in the last three decades [4]. In Ghana, the incidence is within 29-32 per 1000 deliveries [5]. Among developed countries however, up to about $90 \%$ or more EP are diagnosed unruptured but in developing countries diagnoses of unruptured cases are rare. For instance in Ghana the diagnoses of unruptured EP's since early 1990`s has remained in the rates of 1.9\% to $8.5 \%$ [3]. This implies that diagnosis prior to rupture is frequently missed.

Despite the abnormality been linked with some considerably risk factors (Pelvic Inflammatory Disease (PID), post abortal \& postpartum sepsis, previous EP, tubal spasm, intra uterine contraceptive device use, ovulation inducing drugs, previous abdominal-pelvic surgeries, emotional and psychological factors) [1,2,5], EP may occur in all races, countries, and in any socio-economic class of women of childbearing age. The theory behind its (EP) occurrence is that, anything that impedes or delays the migration of an embryo to the endometrial cavity is believed to initiate the process [1].

The symptoms of EP are variable but suspicion should heighten if signs of early pregnancy are accompanied by lower abdominal pain, irregular vaginal bleeding, shoulder tip pain and shock combined with episode of collapse. By far the commonest site of abnormality is usually the fallopian tube (about 97\%) though it can also implants in the cervix, abdomen and ovary. The risk of reoccurrence of EP is about $10 \%$ in women with one previous $\mathrm{EP}$ and at least $25 \%$ in women of two or more previous EP. Nonetheless, some patients may not have any risk factors yet develop the abnormality [5]. Furthermore, evidence suggests that about $60 \%$ of women who receive a diagnosis of EP are subsequently able to have intrauterine pregnancy [6].

There is no clear association found to exist between EP and use of oral contraceptives, and uncomplicated miscarriage [5] although age, tobacco smoking and having multiple sexual partners have been known to be minor risk factors [7]. Perhaps there may be more high risk factors associated with EP that have not yet been explored by researchers.
Austin J Obstet Gynecol - Volume 4 Issue 3 - 2017

Submit your Manuscript | www.austinpublishinggroup.com

Asare et al. (C) All rights are reserved
Citation: Asare HK, Waliwu D and Adu-Ntow D. Ruptured Ectopic Pregnancy: A Case of Fair Complexion Woman at Tamale Teaching Hospital, Ghana. Austin J Obstet Gynecol. 2017; 4(3): 1079. 


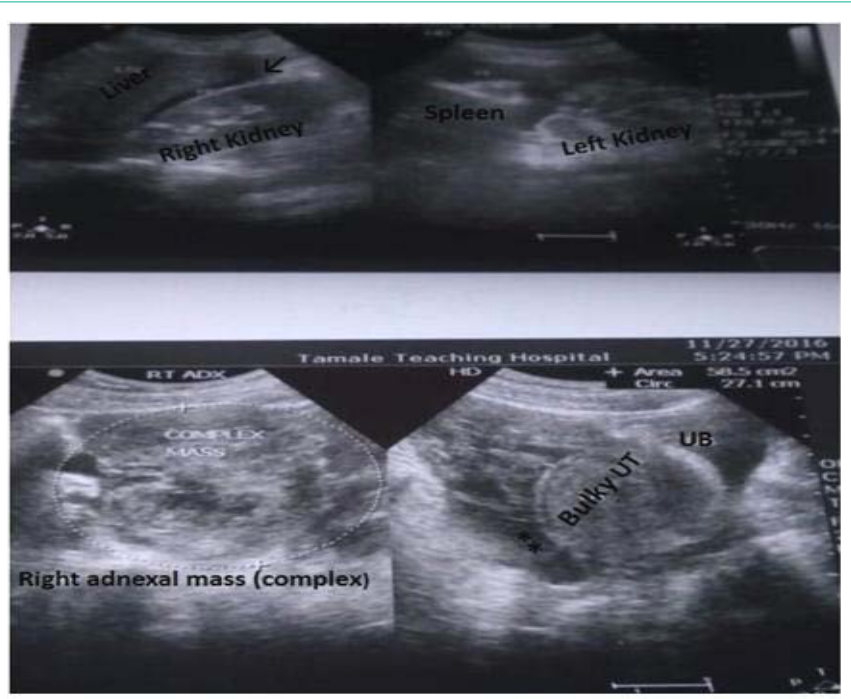

Figure 1: Abdomipelvic ultrasound images showing organs \& features of ruptured ectopic pregnancy UT (uterus), UB (urinary bladder), asterisks ( ${ }^{* *}$ ) is free fluid in clu-de-sac, arrow $(\downarrow)$ pointing towards free fluid in hepato - renal space.

In Opoku et al. study [5] at Komfo Anokye Teaching Hospital in Ghana, the authors established that being fair in skin complexion alone is a predisposed risk of EP among black women of reproductive age independent of any other known major and confounding risk factors $[1,2]$. While our recent case finding agrees largely with their work [5] on grounds that being fair in complexion is a predisposed factor, however, our study does not support the view that fair skin color alone is an autonomous risk factor of EP independent of other confounding factors. In our case, the patient had previous exposure to a minor risk factor (many lifetime partners).

The usage of ultrasound in cases of suspected EP is valuable and efficient with reports estimating detection rates around $77-84.6 \%$ $[2,8]$. Detection may even improve especially if sensitive or high resolution ultrasound technique such as transvaginal scan is used in early stage of pregnancies [2]. Nevertheless, the accuracy of the diagnosis is operator dependent. The sonographic features if present (EP) include; adnexal mass and empty uterus with no gestational sac (except in heterotopic pregnancy). However, if it is a diagnoses of ruptured $\mathrm{EP}$, in addition to the above features there is usually free fluid (hemorrhagic) in the peritoneum spaces.

\section{Conclusion}

The diagnoses of ruptured EP is a case of emergency. Diagnosing EP with ultrasound scan is by far the most common and efficient diagnostic test. Timely laparotomy to aspirate and remove residual conceptus decreases mortality outcomes. It is becoming more apparent that fair skin looking women in Ghana are associating better or consistently with EP. But the diagnosis can be missed if the sonographer or the professional performing the scan is ill-competent since the modality is operator dependent. The finding of this case is overwhelmingly interesting as it compares largely with previous research.

\section{Acknowledgement}

Profound thanks to the woman (patient) and relatives for verbally consenting to publication of the case. Also, appreciation to Dr Nicholas Adzibolosu for his immense support.

\section{References}

1. Dabota BY. Ectopic pregnancy-modern diagnosis and management. Karrava $\mathrm{M}$, editors. In: Management and outcome of ectopic pregnancy in developing countries. Rijeka, Intech. 2011; 109-136.

2. Wakankar R, Kedar K. Ectopic pregnancy- a rising trend. Int J Sci Stud. 2015; 3: 19-22.

3. Nkyekyer K. Ectopic pregnancy in Ghana- time for change. Ghana Med J. 2006; 40: 1-2.

4. Arup KM, Niloptal R, Kakali SK, Pradip KB. Ectopic pregnancy: an analysis of 180 cases. J Indian Med Assoc. 2007; 105: 308-314.

5. Opoku BK, Nguah SB, Azanu W. Ectopic pregnancy: Are fair-colored women at increased risk? Gynecology. 2013; 1-4.

6. Barnhart KT. Ectopic Pregnancy. N Engl J Med. 2009; 361: 379-387.

7. Barnhart KT, Sammel MD, Gracia CR, Chittams J, Hummel AC, Shaunik A, et al. Risk factors for ectopic pregnancy in women with symptomatic first trimester pregnancies. Fertil Steril. 2006; 86: 36-43.

8. Shetty S, Shetty A. A clinical study of ectopic pregnancies in the tertiary care hospital of Mangalore, India. Innov J Med Health Sci. 2014; 4: 305-309.
Austin J Obstet Gynecol - Volume 4 Issue 3 - 2017 Submit your Manuscript | www.austinpublishing group.com Asare et al. () All rights are reserved
Citation: Asare HK, Waliwu D and Adu-Ntow D. Ruptured Ectopic Pregnancy: A Case of Fair Complexion Woman at Tamale Teaching Hospital, Ghana. Austin J Obstet Gynecol. 2017; 4(3): 1079. 